\title{
Remarques sur l'Economie et le Risque
}

\author{
par Georges Martin *
}

C'est un truisme de dire, et le Professeur Nieto l'a confirmé, que le monde est en plein changement, en pleine mutation économique et sociale, ce qui ne peut que rétroagir sur les activités d'assurance.

La haute technologie moderne de plus en plus élaborée, de plus en plus sophistiquée, a complètement modifié les installations industrielles, leur dimension, leur configuration, leur complication et leur potentiel de danger ou de risque. Elle a donné naissance également à toute une série d'activités nouvelles dont l'industrie nucléaire est une des plus caractéristiques, mais on peut en citer bien d'autres : l'aviation, l'industrie chimique, l'industrie pétrolière avec ses problèmes de prospection (plate-formes off shore), de transport (supertankers) et d'exploitation (raffineries, stockage), l'industrie des ordinateurs et la présence de ceux-ci à travers tout le tissu industriel.

Sous la pression de la concurrence mondiale, la recherche du rendement, d'une productivité accrue, a conduit à la concentration, au gigantisme des installations et des entreprises. De nombreux complexes industriels dépassent les 100 millions de dollars, une installation nucléaire atteint le milliard de dollars, l'usine de retraitement de Tricastin, en cours de construction, va coûter 3 milliards de dollars.

Technologie et gigantisme créent de nouveaux défis pour l'assurance. La méconnaissance du risque, faute d'expérience et de statistiques, rend difficile la mesure du risque et donc la définition du juste prix.

L'assureur, et le Professeur Nieto y a fait allusion, doit donc se prémunir contre les écarts. Pour ce faire, il doit disposer de moyens propres et disponibles suffisants à la mesure des risques qu'il prend.

Mais la dimension même des entreprises suscite un autre défi, celui de la capacité mondiale pour les couvrir et donc des moyens financiers pour y faire face.

A côté de ce que les assureurs appellent les grands risques, il en est d'autres : ceux que nous appelons les risques catastrophiques, de nature plus incertaine que les premiers, à faible fréquence et montants élevés, parmi lesquels il faut ranger les catastrophes naturelles, ouragans, inondations, tremblements de terre. Sans doute ont-ils toujours existé, mais la densité d'occupation de notre monde, densité humaine d'abord, mais plus encore densité de valeur des biens qui le couvrent, en accroissent les effets dans des proportions considérables.

* Vice-Président de l'« Association de Genève ». 
Un grand assureur américain, Mr. Roberts, me parlant précisément des tremblements de terre, me disait : imaginons quelle serait la situation des compagnies d'assurance aux Etats-Unis et celle de leurs réassureurs si d'aventure l'un ou l'autre tremblement de terre, qui serait couvert par ces compagnies, se produisait dans l'Ouest, près de San Francisco, ou dans tout autre lieu surpeuplé, tel que Los Angeles ? A côté des dommages directs aux biens, il y aurait aussi à dédommager les nombreuses victimes parmi la population couverte en assurance-vie ainsi que toutes les voitures automobiles détruites. Tout cela mérite réflexion!

Last but not least, la prise de conscience sociale et écologique de nos populations donne une acuité et une extension toutes nouvelles à la notion de responsabilité dans les domaines de la pollution et de la responsabilité des produits. La définition des dommages probables et des primes destinées à les couvrir constitueront dans ce secteur un problème ardu.

Pourtant, me direz-vous, le problème économique fondamental de l'assureur est théoriquement simple, puisqu'il s'agit pour lui d'établir un équilibre permanent entre les primes qu'il perçoit et les sinistres qu'il couvre, compte tenu des écarts qui les grèvent. Mais il s'ajoute ici un nouveau défi, celui de la monnaie et de ses variations. Nous percevons, en effet, une prime à un instant $t$ dans une monnaie déterminée, pour des sinistres, survenant à un instant $t+x$, évalués à ce moment dans une autre unité monétaire et qui seront payés, dans un temps $t+n x$, dans la monnaie du moment et à la valeur du moment.

Et ceci me conduirait à parler du problème de l'inflation. Je l'ai développé voici un an devant les assureurs espagnols à Grenade. A lui tout seul il justifierait tout un débat.

L'inflation est véritablement un des chancres de notre économie puisqu'elle détruit une partie importante de nos actifs laborieusement formés au fil des ans, actifs (fonds d'Etat, obligations, prêts hypothécaires) exprimés en unités monétaires et qui subissent inéluctablement l'érosion de celles-ci.

Il y a là un défi nouveau qui s'ajoute aux autres.

Je me résume : les grands défis auxquels nous avons à faire face maintenant sont : le gigantisme des installations, la technologie moderne qui crée des risques nouveaux, risques nouveaux tels que le nucléaire, la responsabilité produits, la responsabilité pollution, les problèmes de capacité pour couvrir ces risques et par-dessus tout cela l'inflation et son cortège de problèmes subséquents quant à la monnaie, quant aux réserves techniques et aux équilibres essentiels de l'assurance.

Formons le vœu que l'imagination des assureurs et leur savoir faire puissent y faire face. 